\title{
Photophoresis boosts giant planet formation
}

\author{
J. Teiser ${ }^{1}$ and S. E. Dodson-Robinson ${ }^{2}$ \\ 1 Faculty of Physics, University of Duisburg-Essen, Lotharstr. 1, 47048 Duisburg, Germany \\ e-mail: jens.teiser@uni-due.de \\ 2 The University of Texas at Austin, Department of Astronomy, 2515 Speedway Dr. Stop 1400, Austin, TX 78712, USA
}

Received 27 March 2013 / Accepted 17 June 2013

\begin{abstract}
In the core accretion model of giant planet formation, a solid protoplanetary core begins to accrete gas directly from the nebula when its mass reaches $\sim 5 M_{\oplus}$. The protoplanet has at most a few million years to reach runaway gas accretion, as young stars lose their gas disks after 10 million years at the latest. Yet gas accretion also brings small dust grains entrained in the gas into the planetary atmosphere. Dust accretion creates an optically thick protoplanetary atmosphere that cannot efficiently radiate away the kinetic energy deposited by incoming planetesimals. A dust-rich atmosphere severely slows down atmospheric cooling, contraction, and inflow of new gas, in contradiction to the observed timescales of planet formation. Here we show that photophoresis is a strong mechanism for pushing dust out of the planetary atmosphere due to the momentum exchange between gas and dust grains. The thermal radiation from the heated inner atmosphere and core is sufficient to levitate dust grains and to push them outward. Photophoresis can significantly accelerate the formation of giant planets.
\end{abstract}

Key words. planets and satellites: formation - protoplanetary disks - methods: numerical

\section{Introduction}

Up to $15 \%$ of Sunlike stars may host a giant planet consisting of a solid core and a gas envelope (Howard et al. 2012). Such planets require a gaseous environment during their formation, as they must gravitationally attract $\mathrm{H}_{2}$ and $\mathrm{He}$. Giant planet formation must therefore be completed within the lifetime of the gaseous protoplanetary disks, $10 \mathrm{Myr}$ at most (Meyer et al. 2007; Currie et al. 2009).

The planet-metallicity correlation implies that bottom-up growth of giant planets from solid cores, via core accretion, is the dominant planet formation mechanism: planets prefer to orbit stars rich in iron, a major grain-forming material (Gonzalez 1998; Fischer \& Valenti 2005; Neves et al. 2009). Further studies of the composition of planet-host stars show that the efficiency of dust grain nucleation is an important factor in determining whether planets can form (Robinson et al. 2006; Brugamyer et al. 2011). The first step in forming planets by core accretion is the coagulation of small dust grains, which experiments have shown can reach decimeter sizes simply due to van der Waals forces (Blum \& Wurm 2008; Teiser et al. 2011). Dynamical concentration processes such as the streaming instability then allow the dust aggregates to reach large particle densities and form planetesimals (Johansen et al. 2007; Youdin \& Johansen 2007), at which point gravity becomes the dominant force in planet growth.

However, the small grains that are essential building blocks of the giant planets become impediments to planet growth once the planet starts to accrete gas. Small particles $(<\mathrm{mm})$ couple well to the surrounding gas, which increases the opacity of the atmosphere severely (Podolak 2003). The accretion energy produced in the inner part of the growing planet is absorbed by the dust grains, as the atmosphere gets optically thick for thermal radiation. This heats up the system, which directly leads to an increasing gas pressure. This pressure increase slows down the accretion process, as the pressure gradient supports the gas against gravity. Planet formation models have problems reproducing the fast timescales required to explain giant planet formation (Pollack et al. 1996), and those that succeed in forming planets on million-year timescales invoke an arbitrary reduction of the opacity to $2 \%$ of the interstellar value, assuming that grains quickly settle to the sublimation point (Hubickyj et al. 2005; Lissauer et al. 2009; Dodson-Robinson \& Bodenheimer 2010).

There are multiple sources of dust in a protoplanetary atmosphere. Accreted gas brings with it dust agglomerates that are entrained in the gas (Sicilia-Aguilar et al. 2011). These primordial dust particles should already be porous agglomerates, as coagulation of single dust grains to small, highly porous agglomerates is a hit-and stick mechanism which is very fast (Dullemond \& Dominik 2005; Blum \& Wurm 2008). Porous particles have even higher absorbing cross-sections per unit mass than the spheres on which the opacities used by (Pollack et al. 1996) were based, exacerbating the opacity problem. Planetesimals also drift into the planetary core and are exposed to gas drag and to erosion during their way through the planetary atmosphere. This erosion can already start in the outer part of the atmosphere, as gas drag is an efficient erosion mechansim even at low gas pressures (Paraskov et al. 2006). Intense radiation from the inner part of the growing planet can add additional erosion processes, as light induced thermodynamic processes can lead to particle ejection from porous surfaces (Kelling \& Wurm 2011; de Beule et al. 2013). Erosion of planetesimals generates large amounts of small dust aggregates, which are entrained into the planetary atmosphere and can increase the solid/gas ratio and therefore the opacity.

Within this work we present photophoresis as a mechanism capable of pushing small particles outward and clearing the inner parts of the atmosphere from dust grains. Photophoresis occurs when temperature gradients caused by uneven illumination 
of a dust grain lead colliding gas molecules to transfer momentum preferentially to the hotter, more illuminated side of the dust grain. It has already been introduced as an efficient mechanism for material transport in protoplanetary disks (Krauss \& Wurm 2005; Wurm \& Krauss 2006; Wurm \& Haack 2009). Krauss et al. (2007) have also shown that photophoresis is capable of clearing the inner zone of protoplanetary disks of small grains. A growing giant planet is similar to a protoplanetary disk in that in both cases an inner heat source heats a gaseous environment by radiation, producing an outward flux gradient. If photophoresis can eject dust particles from protoplanetary atmospheres faster than they are replenished by accretion, it can drastically reduce the atmospheric accretion timescale and increase the gas/solid mass ratio of the resulting protoplanet (Dodson-Robinson et al. 2008). Here we demonstrate that the dust clearing timescale for two snapshots of growing protoplanetary atmospheres is of order $10^{3}-10^{4}$ years, far quicker than the planet growth timescale.

In Sect. 2, we discuss the model protoplanetary atmospheres used for the photophoresis calculation. The principle of photophoresis and the important parameters which determine its efficiency are presented in Sect. 3. In Sect. 4 we describe the resulting drift timescales from our photophoresis model. In Sect. 5, we discuss the efficiency of photophoresis compared with other dust-clearing mechanisms and present evidence that agglomerates in the protoplanetary atmosphere should be porous, increasing the photophoretic force. We present our conclusions in Sect. 6.

\section{Model}

The growth of a protoplanet can be divided into three phases: (1) planetesimal accretion that builds up a solid core; (2) slow gas and planetesimal accretion that builds the protoplanetary atmosphere at a nearly constant rate; and (3) runaway growth that takes the protoplanet from ice-giant mass $\left(\sim 15 M_{\oplus}\right)$ to gas-giant mass in 1000 years (Pollack et al. 1996; Hubickyj et al. 2005). It is Phase 2 which concerns us here - an epoch where cooling and contraction of the protoplanetary atmosphere is limited by continual planetesimal heating and high opacity due to small grains, preventing new gas from entering the protoplanet's Hill sphere.

Here we present a proof-of-concept that photophoresis can affect the thermal balance of a protoplanetary atmosphere by calculating the photophoretic force in two Phase 2 time snapshots from the Uranus and Neptune growth models of DodsonRobinson \& Bodenheimer (2010) for a protoplanet at $15 \mathrm{AU}$ from the Sun. We use the planet-growth models as standalone input into the photophoresis calculation, which was not incorporated into the atmospheric growth model.

In each time snapshot, the protoplanet atmosphere fills the smaller of the Hill sphere, $R_{\mathrm{h}}=a\left[M_{\text {planet }} /\left(3 M_{*}\right)\right]^{1 / 3}$ (where $a$ is the semimajor axis of the protoplanet's orbit, Armitage 2010), or the Bondi sphere, $R_{\mathrm{acc}}=G M_{\text {planet }} / c_{\mathrm{s}}^{2}$ (where $c_{\mathrm{s}}$ is the sound speed of the surrounding nebula, Bondi 1952). The protoplanet is accreting $100-\mathrm{km}$ planetesimals at a rate of

$\dot{M}_{\text {solid }}=C_{1} \pi \Sigma_{\text {solid }} R_{\mathrm{c}} R_{\mathrm{h}} \Omega$,

where $\Sigma_{\text {solid }}$ is the surface density of planetesimals in the disk, $R_{\mathrm{c}} \approx 0.01 R_{\mathrm{h}}$ is the effective capture radius of the protoplanet, which is well within the range found in literature (Kary et al. 1993). $\Omega$ is the Keplerian angular speed and $C_{1}$ is a constant near unity (Papaloizou \& Terquem 1999). Planetesimals enter the protoplanet atmosphere at the Hill velocity, $v_{\mathrm{h}}=R_{\mathrm{h}} \Omega$, and

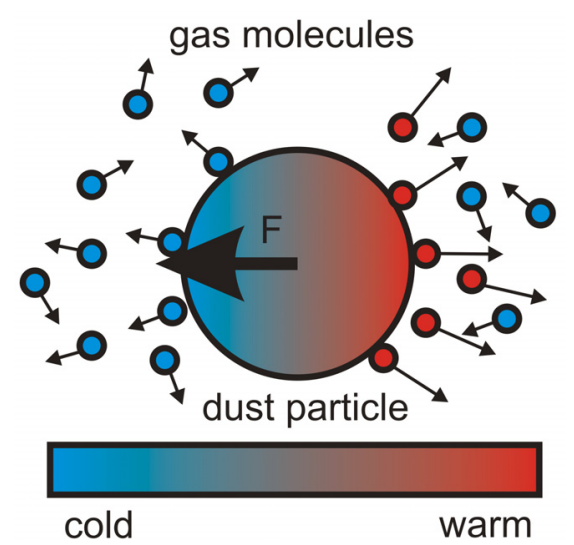

Fig. 1. Schematic sketch of the principle of photophoresis. The illuminated surface heats up and transfers momentum to gas molecules, which finally leads to a net force in the direction of light.

begin to deposit their kinetic energy in each layer of the atmosphere by gas drag heating, dissipation of the kinetic energy of ablated material, and sinking of material ablated in layers above. Energy is also removed from the atmosphere due to latent heat as planetesimal material vaporizes. For full details of the energy calculation, see Pollack et al. (1996). A planetesimal is considered captured if it displaces an amount of gaseous material equal to its own mass or deposits $50 \%$ or more of its mass into the atmosphere. Once $50 \%$ of the planetesimal mass has been ablated, we invoke the sinking approximation so that the rest of the planetesimal and the ablated debris sink rapidly to the core (Hubickyj et al. 2005).

We use a Henyey-type code to solve the standard equations of stellar structure for the protoplanetary atmosphere (Henyey et al. 1964). Opacities are modified from the standard interstellar case according to the grain growth and settling prescription of Podolak (2003). New gas enters the atmosphere due to the combined effects of cooling-induced contraction and expansion of the Hill sphere due to accretion. During each timestep of the planet-growth simulation, the atmosphere is assumed to be in hydrostatic equilibrium. The first snapshot selected for the photophoresis study (Snapshot 1) has atmospheric mass $M_{\mathrm{atm}}=0.11 M_{\oplus}$ and core mass $M_{\text {core }}=4.39 M_{\oplus}$. Snapshot 2 has $M_{\mathrm{atm}}=0.3 M_{\oplus}$ and $M_{\text {core }}=7.48 M_{\oplus}$.

\section{Photophoresis}

Photophoresis is an interaction between particles and the surrounding gas in a medium with non-isotropic illumination. The illuminated side of a particle is heated as the particle absorbs light. The other side stays cooler and a temperature gradient forms along the particle surface. A temperature gradient forms for any kind of radiation which is absorbed by the particle (optical or thermal infrared). Gas molecules accommodate to the particle surface and adopt the surface temperature where they collide with a grain. Such collisions change the mean velocity of the molecules, as the mean thermal velocity depends on grain temperature as $T_{\text {grain }}^{1 / 2}$. On the warm side of a grain, colliding gas molecules gain more momentum than on the cooler side. The velocity difference leads to a net momentum transfer from the gas molecules to the particle, which amounts to a net force in the direction of the temperature gradient (direction of light flux). The principle of photophoresis is visualized in Fig. 1.

Photophoresis is a thermodynamic non-equilibrium process and depends on many particle and gas parameters, especially the 
gas pressure. It reaches the maximum force $F_{\max }$ at the pressure $p_{\max }$, at which the mean free path of the gas molecules is of the same order of magnitude as the particle size. A semiempirical description of the photophoretic force $F_{\mathrm{ph}}$ and the influence of the thermodynamic parameters and particle size is given by Rohatschek (1995):

$$
\begin{aligned}
F_{\mathrm{ph}} & =2 F_{\max }\left(\frac{p_{\mathrm{max}}}{p}+\frac{p}{p_{\mathrm{max}}}\right)^{-1} \\
p_{\max } & =\frac{\eta}{r} \sqrt{\frac{12 R_{\mathrm{gas}} T}{M_{\mathrm{mol}}}} \\
F_{\text {max }} & =\frac{\pi \eta r^{2} I}{2 k_{\mathrm{th}}} \sqrt{\frac{R_{\mathrm{gas}}}{3 T M_{\mathrm{mol}}}} .
\end{aligned}
$$

Here, $r$ is the particle radius, $\eta=1.8 \times 10^{-5} \mathrm{~Pa}$ is the dynamic gas viscosity, $k_{\text {th }}$ is the thermal conductivity of the dust, $I$ is the incoming irradiation, $T$ is the gas temperature, $M_{\mathrm{mol}}$ is the molar mass of the gas $(2.34 \mathrm{~g} / \mathrm{mol}$ for a mixture of mostly hydrogen and helium), and $R_{\text {gas }}=8.31 \mathrm{~J} /(\mathrm{mol} \mathrm{K})$ is the gas constant. Rohatschek (1995) uses an additional thermal creep parameter in the model which was set to 1.14 . This is not necessarily the case for real particles but to compare experimental results with the semi-analytical solution by Rohatschek (1995) it is set to 1 for simplicity in this work.

The planetary atmospheres calculated in the models used here cover a wide pressure range from several bar in the inner atmosphere to mbar in the outer atmosphere. Although the pressures in the inner part are much larger than the optimal pressure for photophoresis, the radiation flux from the inner towards the outer parts is so large that photophoresis has to be considered. To calculate the photophoretic force we assume spherical dust agglomerates with a radius of $r=10 \mu \mathrm{m}$, thermal conductivity of $k_{\mathrm{th}}=0.1 \mathrm{~W} \mathrm{~K}^{-1} \mathrm{~m}^{-1}$, and a mean density of $2000 \mathrm{~kg} / \mathrm{m}^{3}$. This density corresponds to an olivine agglomerates with a porosity of the order of $55 \%$, which is still very compact in comparison to dust agglomerates formed by coagulation processes (Blum \& Wurm 2008). For highly porous quartz agglomerates thermal conductivities of $k_{\text {th }} \approx 0.01 \mathrm{~W} / \mathrm{Km}$ were found by Krause et al. (2011) for similar porosities. We take a rather conservative value of $k_{\text {th }}$ so as not to overestimate $F_{\max }$. Our grain size of $r=10 \mu \mathrm{m}$, which is in the range of grain sizes determined for comets (Harker et al. 2002), is also conservative as $p_{\max }$ increases with decreasing particle size. All other parameters (light flux, pressure, temperature) are taken from the formation model described in Sect. 2.

To evaluate the efficiency of photophoresis as a transport mechanism we calculated the force ratio between photophoresis and gravity for Snapshots 1 and 2. As long as the ratio between the two forces is larger than 1, particles drift outward until they reach the point of equilibrium. However, not only the force ratio is of great interest, but also the time the particles need to reach the point of equilibrium. We assumed that solid particles exist as soon as the temperature is below $1800 \mathrm{~K}$ and calculated the drift time they need to reach the point of equilibrium.

Particles are accelerated by the acting resulting force $F_{\text {res }}=$ $F_{\text {ph }}-F_{\text {grav }}$ with $F_{\text {grav }}=\gamma m_{\text {dust }} m_{\text {in }} r^{-2}$ with the gravity constant $\gamma=6.674^{-11} \mathrm{kgm}^{-3}$ and $m_{\text {in }}$ being the total mass of the core and the atmosphere within the radius $r$. Particles are decelerated by gas drag, which leads to a constant drift velocity of

$v_{\mathrm{drift}}=a_{\mathrm{res}} \tau$.

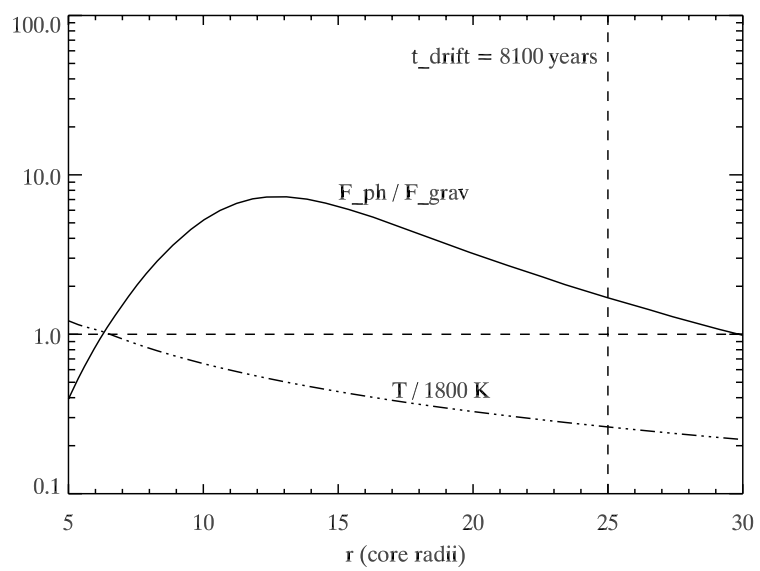

Fig. 2. Photophoretic strength and temperature distribution for a growing giant planet with a total mass (core+atmosphere) of 4.5 earth masses and a core radius of 1.64 earth radii.

Here, $a_{\text {res }}$ is the resulting acceleration $\left(F_{\text {res }} / m_{\text {dust }}\right)$ and $\tau$ is the gas coupling time of the dust grains. The gas coupling behaviour of the dust grains depends on the pressure. Hydrodynamical processes depend on the Knudsen number, which is defined as $K n=\lambda / r_{\text {dust }}$ with the mean free path $\lambda$ and the particle radius $r_{\text {dust }}$. To determine the gas coupling time for $K n>10$ we use the gas drag of free molecular flow regime, which leads to

$\tau=0.68 \frac{m_{\text {dust }}}{\pi r_{\text {dust }}^{2} \rho_{\text {gas }} v_{\text {gas }}}=0.91 \frac{r_{\text {dust }} \rho_{\text {dust }}}{\rho_{\text {gas }} \nu_{\text {gas }}}$.

Here, $\rho_{\text {gas }}$ is the density and $v_{\text {gas }}$ is the thermal velocity of the gas molecules, which can be written as $v_{\mathrm{gas}}=\sqrt{8 R_{\mathrm{gas}} T /\left(\pi M_{\mathrm{mol}}\right)}$ with the mass of a dust grain $m_{\text {dust }}$. The gas coupling time for $K n<0.1$ is determined by the Stokes friction and is given as

$\tau=\frac{m_{\text {dust }}}{6 \pi r_{\text {dust }} \eta}=\frac{2 r_{\text {dust }}^{2} \rho_{\text {dust }}}{9 \eta}$

Stokes friction can be applied since the Reynolds numbers for dust particles are well below $R e=1$, even for the large densities in the inner atmosphere. For the transition regime with $0.1 \leq$ $K n \leq 10$ we use Stokes friction with the Cunningham correction (Cunningham 1910; Hutchins et al. 1995), which is given as

$\tau=\frac{m_{\text {dust }}}{6 \pi r_{\text {dust }} \eta} \cdot\left(1+K n \cdot\left(1.231+0.47 \mathrm{e}^{-1.178 / K n}\right)\right)$.

With this set of equations the coupling times and therefore also the drift velocities can be calculated for dust grains moving outward, which also gives the drifting timescales for the dust grains.

\section{Results}

The accretion rate and therefore also the heat production in the inner part of the growing atmosphere change during the formation of giant planets. Within this work the influence of photophoresis on the evolution of giant planets is studied for a protoplanet at $15 \mathrm{AU}$ solar distance at two different evolutionary stages. Snapshot 1 presents the growing planet after a formation time of 3.07 million years with a planetary core of 4.39 earth masses and an atmosphere of 0.11 earth masses. The result is shown in Fig. 2.

Dust particles only exist in the regions of the planetary atmosphere, in which the temperature (dash-dotted curve, normalized to $1800 \mathrm{~K}$ ) is below the condensation temperature (we assumed 


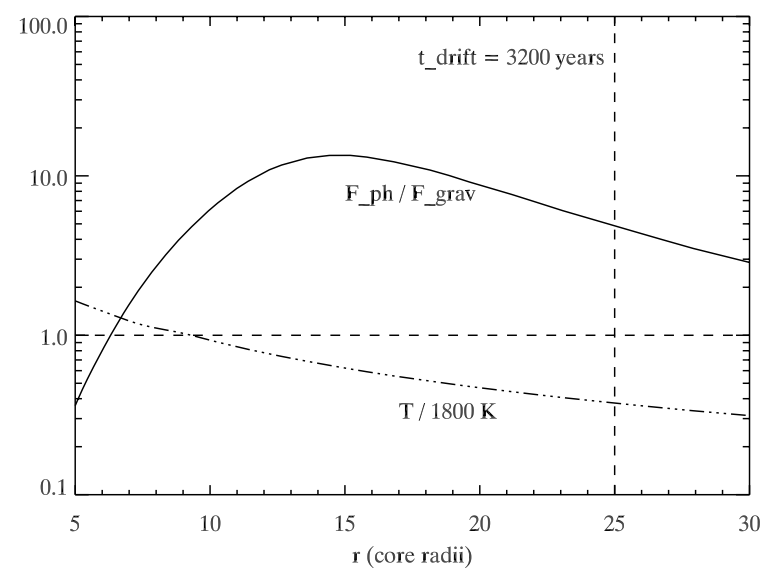

Fig. 3. Photophoretic strength and temperature distribution for a growing giant planet with a core of 7.8 earth masses and a core radius of 1.96 earth radii.

$1800 \mathrm{~K}$, although in reality dust grains condense over a range of approximately $1200 \mathrm{~K}-2100 \mathrm{~K}$ ). In Snapshot 1, dust grains begin to condense at a radial distance from the center of the protoplanet of 6.4 core radii. At this point the photophoretic force (solid curve, normalized by gravity) is larger than gravity by a factor of 1.1. The point of equilibrium, for which the photophoretic force exactly balances gravity, is at a radial distance of 30 core radii. A dust grain with a radius of $r=10 \mu \mathrm{m}$ needs $3 \times 10^{4}$ years to drift the distance between those points. It is important to note that the drift process takes longest in the outer part of the atmosphere, as it takes only 8100 years for the same dust grain to reach a radial distance of 25 core radii (see Fig. 2).

In Snapshot 2, a later growth stage, the growing planet has accreted much more mass and more accretion heat has developed in the inner part, so temperatures increase and photophoresis gets stronger. The result is shown in Fig. 3 for a planetary core of 7.48 earth masses and an atmosphere of 0.3 earth masses with a formation time of 4.39 million years. The region in which dust grains exist has moved outward to a radial distance of 8.8 core radii, while the point of equilibrium between photophoresis and gravity has moved to 42 core radii. At the inner edge of the dusty part of the atmosphere photophoresis exceeds gravity by a factor of 4 , which leads to drift timescales of $3.3 \times 10^{4}$ years from this point to the point of equilibrium. Here, it takes only $3.2 \times 10^{3}$ years for the same dust agglomerate to reach a radial distance of 25 core radii. In both snapshots photophoresis is capable of moving dust outward.

\section{Discussion}

An additional radiation-based process is radiation pressure, which is also capable of accelerating particles. Radiation pressure is the momentum transfer from photons on a particle and can be written as $F_{\text {rad }}=r^{2} I / c$ for a spherical, absorbing particle, with the intensity $I$, light speed $c$ and the particle radius $r$. Radiation pressure plays a significant role in the solar system, as comet tails and asteroids are strongly influenced by it or by related processes (Poynting-Robertson Effect, Yarkowsky Effect). For gaseous environments like the solar nebula or protoplanetary atmospheres, the photophoretic force exceeds radiation pressure by orders of magnitude. For both snapshots (Figs. 2 and 3) photophoresis is larger than radiation pressure by at least three orders of magnitude for the dust-rich part of the planetary atmosphere.
In Eq. (4) it is shown that photophoresis scales directly with the intensity of the light flux. This parameter varies strongly with the radial distance to the planetary core and depending on the planet formation scenario. In case of the second snapshot (Fig. 3) an intensity reduced by a factor of 10 will reduce the zone in which photophoresis exceeds gravity to a radial distance from the core between 12 and 19 core radii. In case of an intensity by a factor of 10 larger than in Fig. 3, this zone extends from the direct core environment to a distance of 90 core radii instead of 42 core radii as in Fig. 3.

The presented results show that photophoresis can indeed be an important process for the formation of giant planets. Depending on the evolutionary state of the growing planet, photophoresis can exceed gravity by a factor of 10 , leading to an outward drift of dust grains. The timescales for this drift process are small in comparison to the formation timescales of giant planets. The gas pressure within the inner part of the planetary atmosphere is much larger than the pressure for which photophoresis is most efficient. The photophoretic efficiency will therefore increase with the growing distance to the planetary core or the grain-sublimation line.

The light intensity drops steeply with the radial distance, which leads to decreasing photophoresis, especially as dust in the planetary atmosphere absorbs and scatters the radiation from the innermost atmosphere, enhancing the decrease. However, as dust grains drift outward due to photophoresis, the zone where $F_{\text {ph }}>F_{\text {grav }}$ is quickly cleared from absorbing or scattering dust grains. This clearing leads to an even stronger effect of photophoresis, as the radiation from the inner parts can then reach the dust grains directly. A similar mechanism has already been described for the inner zone of protoplanetary disks by Krauss et al. (2007). The consequence of this mechanism is a kind of runaway process, as the clearing of the inner zone enhances photophoresis in the outer parts of the planetary atmosphere. This feedback reaction is not treated in the snapshots shown in Figs. 2 and 3 , as a revision of the complete formation model is beyond the scope of this work. In future work we will include photophoresis in the atmospheric opacity calculations, so the atmospheric accretion rate and photophoretic force can be calculated self-consistently.

The material properties of the dust agglomerates in the planetary atmosphere are based on simplified assumptions concerning density, thermal conductivity, and porosity. It is important to note that these properties have a strong influence on the photophoretic acceleration. The exact properties of the dust grains are unknown. Bodies produced by coagulation processes are highly porous ( $\geq 60 \%$ porosity), even if the collision velocities reach values of $60 \mathrm{~m} / \mathrm{s}$ (Teiser \& Wurm 2009; Meisner et al. 2012). Planetesimals, which are not thermally processed, are still highly porous. Fragments which are generated by erosion processes will therefore also be highly porous. Primordial dust generated by condensation in protoplanetary disks is initially micron-sized during the early formation phase of the disks. Micron-sized particles coagulate very efficiently, as collision velocities are small and cohesion forces are large. Efficient coagulation has been demonstrated both theoretically and experimentally (Krause \& Blum 2004; Wada et al. 2009). Single monomers do therefore not exist after very short timescales due to this fast coagulation (Dullemond \& Dominik 2005). In a more complete calculation of the photophoretic force, dust particles should be treated as highly porous agglomerates rather than spherical, porous monomers.

The thermal properties of porous agglomerates are strongly influenced by the porosity. With a porosity of $55 \%$ and 
$k_{\mathrm{th}}=0.1 \mathrm{~W} / \mathrm{Km}$ we considered rather small porosities and large thermal conductivities in comparison to literature (Meisner et al. 2012; Krause et al. 2011). The photophoretic force scales with $1 / k_{\text {th }}$, so a greater porosity leads directly to a smaller thermal conductivity and therefore to an increased photohoretic force. The reduced density automatically leads to a larger acceleration due to smaller inertia. In this case the clearing process will be enhanced dramatically. Of course, for perfectly solid, non-porous monomers the clearing efficiency will be reduced and photophoresis will not exceed gravity in the inner part of the atmosphere. However, it is questionable if such solid particles exist in large amounts in the outer part of the solar system. Solid particles of mm-size, like e.g. chondrules, usually are thermally processed (Scott 2007), which is assumed to happen in the inner part of the solar system. Bodies in the outer solar system typically contain large amounts of ice and are assumed to be highly porous. We conclude that the majority of the small particles in the atmosphere of growing giant planets consists of porous dust agglomerates with very small thermal conductivities.

Mutual collisions between dust agglomerates might lead to a fragmentation cascade which leads to a wide size distribution. In both snapshots photophoresis is capable of transporting material of a certain size distribution, but the timescales vary significantly with the particle size. In the first snapshot (Fig. 2) the drift timescale to reach the point of equilibrium increases to $2.6 \times 10^{5}$ for a particle radius of $1 \mu \mathrm{m}$. Additionally, the starting point, for which photophoresis exceeds gravity also varies, as the photophoretic force reaches its maximum at different pressures depending on the particle size. With increasing particle size this thresholds is found at larger distances from the planetary core, which means that for $100 \mu \mathrm{m}$ particles this mechanism could only work at a radial distance between 23 core radii and 25 core radii. In the second snapshot (Fig. 3) photophoresis exceeds gravity in the complete inner part of the dust rich atmosphere for particle sizes up to $200 \mu \mathrm{m}$. However, the timescales still vary from $t=3 \times 10^{5}$ years for a particle radius of $1 \mu \mathrm{m}$ to $t=7 \times 10^{3}$ years for a particle radius of $100 \mu \mathrm{m}$.

Photophoresis is based on the non-symmetric heating of particles, as a temperature gradient along the surface has to form. In case of fast rotation particles are heated more homogeneously, which reduces the temperature gradient. In case of planetary atmospheres the gas pressure is high, which leads to a strong damping of any rotation. Additionally, experiments have shown that particles tend to align their rotation axis parallel to the light flux, if they are subject to photophoresis and gravity (van Eymeren \& Wurm 2012). A temperature gradient in the direction of the light flux is therefore not prevented. Both processes lead to the conclusion that rotation does not prevent photophoretic motion and can be neglected in simplified calculations as this.

\section{Summary and conclusions}

The formation of giant planets has to happen fast, as their formation has to be completed within the lifetime of the protoplanetary disk. Small particles in the planetary atmosphere reduce the formation speed, as accretion-produced heat cannot be efficiently transported outward by radiation. Photophoresis can push small particles to the outer parts of the planetary atmosphere, clearing the inner parts so accretion heat can be quickly transported outward. In the cleared zone no absorption takes place, so the light flux in the outer regions increases as well. Photophoretic clearing therefore leads directly to a runaway process, in which photophoresis amplifies itself by clearing the inner parts of the atmosphere.

As the heat is transported outward, the forming protoplanet begins to cool and contract efficiency, allowing new gas to enter the Hill sphere. Photophoresis therefore increases the possible gas accretion rate and may lead to a substantial speedup in giant planet formation. Dodson-Robinson et al. (2008) demonstrated that a low-opacity atmosphere increases the gas/solid mass ratio of the newly formed planet, so photophoresis may modify planetary structure as well as growth timescale. In future work we will present updated planet-growth calculations that self-consistently incorporate photophoresis into the planetary atmosphere calculation.

Acknowledgements. We thank Peter Bodenheimer for the collaboration and for providing access to the planet formation model. Funding for S.D.R.'s work was provided by National Science Foundation CAREER award AST-1055910.

\section{References}

Armitage, P. J. 2010, Astrophysics of Planet Formation (Cambridge, UK: Cambridge University Press), 294

Bondi, H. 1952, MNRAS, 112, 195

Blum, J., \& Wurm, G. 2008, ARA\&A, 46, 21

Brugamyer, E., Dodson-Robinson, S. E., Cochran, W. D., \& Sneden, C. S. 2011, ApJ, 738, 97

Cunningham, E. 1910, Proc. R. Soc., 83, 357

Currie, T., Lada, C. J., Plavchan, P., et al. 2009, ApJ, 698, 1

de Beule, C., Kelling, T., Wurm, G., Teiser, J., \& Jankowski, T. 2013, ApJ, 763,

Dodson-Robinson, S. E., \& Bodenheimer, P. 2010, Icarus, 207, 491

Dodson-Robinson, S. E., Bodenheimer, P., Laughlin, G., et al. 2008, ApJ, 688, 99

Dullemond, C. P., \& Dominik, C. 2005, A\&A, 434, 971

Fischer, D. A., \& Valenti, J. 2005, ApJ, 622, 1102

Gonzalez, G. 1998, A\&A, 334, 221

Harker, D. E., Wooden, D. H., Woodward, C. E., \& Lisse, C. M. 2002, ApJ, 580, 579

Henyey, L. G., Forbes, J. E., \& Gould, N. L. 1964, ApJ, 139, 306

Howard, A. W., Marcy, G. W., Bryson, S. T., et al. 2012, ApJS, 201, 15

Hubickyj, O., Bodenheimer, P., \& Lissauer, J. J. 2005, Icarus, 179, 415

Hutchins, D. K., Harper, M. H., \& Felder, R. L. 1995, Aerosol. Sci. Tech., 22, 202

Johansen, A., Oishi, J. S., Mac Low, M.-M., et al. 2007, Nature, 448, 1022

Kary, D. M., Lissauer, J. J., \& Greenzweig, Y. 1993, Icarus, 106, 288

Kelling, T., \& Wurm, G. 2011, ApJ, 733, 120

Krause, M., \& Blum, J. 2004, Phys. Rev. Lett., 93, 021103

Krause, M., Blum, J., Skorov, Y. V., \& Trieloff, M. 2011, Icarus, 214, 286

Krauss, O., \& Wurm, G. 2005, ApJ, 630, 1088

Krauss, O., Wurm, G., Mousis, O., et al. 2007, A\&A, 462, 977

Lissauer, J. J., Hubickyj, O., D’Angelo, G., \& Bodenheimer, P. 2009, Icarus, 199,338

Meisner, T., Wurm, G., \& Teiser, J. 2012, A\&A, 544, A138

Meyer, M. R., Backman, D. E., Weinberger, A. J., \& Wyatt, M. C. 2007, in Protostars and Planets V, eds. B. Reipurth, D. Jewitt, \& K. Keil, 573

Neves, V., Santos, N. C., Sousa, S. G., Correia, A. C. M., \& Israelian, G. 2009, A\&A, 497, 563

Papaloizou, J. C. B., \& Terquem, C. 1999, ApJ, 521, 823

Paraskov, G. B., Wurm, G., \& Krauss, O. 2006, ApJ, 648, 1219

Podolak, M. 2003, Icarus, 165, 428

Pollack, J. B., Hubickyj, O., Bodenheimer, P., et al. 1996, Icarus, 124, 62

Robinson, S. E., Laughlin, G. P., Bodenheimer, P., \& Fischer, D. A. 2006, ApJ, 643, 484

Rohatschek, H. 1995, J. Aerosol Sci., 26, 717

Scott, E. R. D. 2007, Ann. Rev. Earth Planet. Sci., 35, 577

Sicilia-Aguilar, A., Henning, T., Dullemond, C. P., et al. 2011, ApJ, 742, 39

Teiser, J., \& Wurm, G. 2009, MNRAS, 393, 1584

Teiser, J., Engelhardt, I., \& Wurm, G. 2011, ApJ, 742, 5

van Eymeren, J., \& Wurm, G. 2012, MNRAS, 420, 183

Wada, K., Tanaka, H., Suyama, T., Kimura, H., \& Yamamoto, T. 2009, ApJ, 702, 1490

Wurm, G., \& Krauss, O. 2006, Icarus, 180, 487

Wurm, G., \& Haack, H. 2009, Meteorit. Planet. Sci., 44, 689

Youdin, A., \& Johansen, A. 2007, ApJ, 662, 613 